\title{
TIME HARMONIC ELECTROMAGNETIC FIELDS IN AN BIAXIAL ANISOTROPIC MEDIUM
}

\author{
J. Sheen \\ Department of Communication Engineering \\ Oriental Institute of Technology \\ 58, Sec. 2, Sze-Chuan Rd., Pan-Chiao City 220, Taiwan
}

\begin{abstract}
This study develops the scalar wave equations of auxiliary vector potentials for time harmonic electric and magnetic fields on transverse electric, transverse magnetic and transverse electromagnetic modes in an biaxial anisotropic medium. The concise scalar wave equations are achieved in both rectangular and cylindrical coordinates. Moreover, in an biaxial medium, it is identified that the decoupled TE, TM, and TEM modes can only exist under certain conditions for various modes respectively which clarify the suspect about the existence of these modes that was reported previously.
\end{abstract}

\section{Introduction}

\section{Derivation of Scalar Wave Equations}

\section{Summary}

\section{Appendix A.}

\section{Acknowledgment}

\section{References}

\section{INTRODUCTION}

The analysis of electromagnetic field for anisotropic object is an important issue. Work has been conducted on various areas [1-14]. It is recognized that the widely used substrate material sapphire is anisotropic. The lithium niobate $\left(\mathrm{LiNbO}_{3}\right)$ is used in the design of integrated optics devices and has an uniaxial permittivity tensor. Therefore, analyses for devices based on these materials must account for the possibility of anisotropic material tensor. 
For the electromagnetic boundary, the most widely known field configurations are those referred to as the transverse electric (TE), transverse magnetic (TM), and transverse electromagnetic (TEM) modes. Work on the electromagnetic fields of these modes for an biaxial anisotropic medium remains incomplete. Moreover, it was reported $[3,4]$ that the biaxial case does not allow the decomposition of field into TE and TM modes.

A different aspect was reached in this work. The TE, TM, and TEM modes do exist but only under especial conditions. The completed expressions for these modes will be given. The required condition for each mode to exist will be presented.

The auxiliary vector potentials are well known to be $F$ and $A$, where

$$
\begin{aligned}
& D=-\nabla \times F \\
& B=\nabla \times A
\end{aligned}
$$

can be used to solve the electric and magnetic fields of the TE, TM, and TEM modes in an isotropic medium, and can be found in some electromagnetics books [15]. This study develops the scalar wave equations of $A$ or $F$ for TE, TM, and TEM modes transverse to various directions $\left(\mathrm{TE}^{x, y, z}, \mathrm{TM}^{x, y, z}\right.$, and $\mathrm{TEM}^{x, y, z}$ ) in both rectangular and cylindrical coordinates in an biaxial anisotropic medium. The electric and magnetic fields in an anisotropic medium with various geometries can then be obtained by solving the scalar wave equations with adequate boundary conditions. The derivation procedures are shown to be quite simple. Since the individual procedures for different modes are similar, this study only presents detailed procedures for the first derived $\mathrm{TE}^{y}$ mode.

\section{DERIVATION OF SCALAR WAVE EQUATIONS}

The work is focused on the biaxial anisotropic medium, where the permittivity and permeability are

$$
\varepsilon=\left[\begin{array}{ccc}
\varepsilon_{x} & 0 & 0 \\
0 & \varepsilon_{y} & 0 \\
0 & 0 & \varepsilon_{z}
\end{array}\right] \text { and } \mu=\left[\begin{array}{ccc}
\mu_{x} & 0 & 0 \\
0 & \mu_{y} & 0 \\
0 & 0 & \mu_{z}
\end{array}\right]
$$

respectively. Therefore,

$$
\begin{aligned}
& D=\hat{a}_{x} D_{x}+\hat{a}_{y} D_{y}+\hat{a}_{z} D_{z}=\hat{a}_{x} \varepsilon_{x} E_{x}+\hat{a}_{y} \varepsilon_{y} E_{y}+\hat{a}_{z} \varepsilon_{z} E_{z} \\
& B=\hat{a}_{x} B_{x}+\hat{a}_{y} B_{y}+\hat{a}_{z} B_{z}=\hat{a}_{x} \mu_{x} H_{x}+\hat{a}_{y} \mu_{y} H_{y}+\hat{a}_{z} \mu_{z} H_{z}
\end{aligned}
$$


for rectangular coordinate, and

$$
\begin{aligned}
& D=\hat{a}_{r} D_{r}+\hat{a}_{\phi} D_{\phi}+\hat{a}_{z} D_{z}=\hat{a}_{r} \varepsilon_{r} E_{r}+\hat{a}_{\phi} \varepsilon_{\phi} E_{\phi}+\hat{a}_{z} \varepsilon_{z} E_{z} \\
& B=\hat{a}_{r} B_{r}+\hat{a}_{\phi} B_{\phi}+\hat{a}_{z} B_{z}=\hat{a}_{r} \mu_{r} H_{r}+\hat{a}_{\phi} \mu_{\phi} H_{\phi}+\hat{a}_{z} \mu_{z} H_{z}
\end{aligned}
$$

for cylindrical coordinate, where

$$
\begin{aligned}
& \frac{(\varepsilon, \mu)_{r}}{(\varepsilon, \mu)_{x}} \cos ^{2} \phi+\frac{(\varepsilon, \mu)_{r}}{(\varepsilon, \mu)_{y}} \sin ^{2} \phi=1 \\
& \frac{(\varepsilon, \mu)_{\phi}}{(\varepsilon, \mu)_{x}} \sin ^{2} \phi+\frac{(\varepsilon, \mu)_{\phi}}{(\varepsilon, \mu)_{y}} \cos ^{2} \phi=1
\end{aligned}
$$

Source free and time harmonic will be assumed in the following derivations.

\section{(A) TE Modes}

The rectangular coordinate is firstly considered and starts with the $\mathrm{TE}$ to the $y$ direction ( $\mathrm{TE}^{y}$ mode). For $\mathrm{TE}^{y}$ mode, let

$$
F=\hat{a}_{y} F_{y}(x, y, z) ; \quad F_{x}=F_{z}=0
$$

From equation (1),

$$
E_{x}=\frac{1}{\varepsilon_{x}} \frac{\partial F_{y}}{\partial z} ; \quad E_{y}=0 ; \quad E_{z}=-\frac{1}{\varepsilon_{z}} \frac{\partial F_{y}}{\partial x}
$$

From equation (4) and Maxwell's equation

$$
\nabla \times E=-j \omega B
$$

we can get,

$$
\frac{\partial E_{z}}{\partial y}=-j \omega \mu_{x} H_{x} ; \quad \frac{\partial E_{x}}{\partial z}-\frac{\partial E_{z}}{\partial x}=-j \omega \mu_{y} H_{y} ; \quad \frac{\partial E_{x}}{\partial y}=j \omega \mu_{z} H_{z}
$$

In addition, from equation (1),

$$
\nabla \times D=\nabla^{2} F-\nabla \nabla \cdot F
$$

and by adding the following equation,

$$
H=-\nabla \phi_{m}-j \omega F
$$


where $\phi_{m}$ represents a magnetic scalar potential. We have

$$
\begin{aligned}
\nabla \times D= & \hat{a}_{x} \varepsilon_{z} \frac{\partial E_{z}}{\partial y}+\hat{a}_{y}\left(\varepsilon_{x} \frac{\partial E_{x}}{\partial z}-\varepsilon_{z} \frac{\partial E_{z}}{\partial x}\right)-\hat{a}_{z} \varepsilon_{x} \frac{\partial E_{x}}{\partial y} \\
= & -\hat{a}_{x} j \omega \mu_{x} \varepsilon_{z} H_{x}+\hat{a}_{y}\left[-j \omega \mu_{y} \varepsilon_{x} H_{y}+\left(\varepsilon_{x}-\varepsilon_{z}\right) \frac{\partial E_{z}}{\partial x}\right]-\hat{a}_{z} j \omega \mu_{z} \varepsilon_{x} H_{z} \\
= & \hat{a}_{x} j \omega \mu_{x} \varepsilon_{z} \frac{\partial \phi_{m}}{\partial x}+\hat{a}_{y}\left[j \omega \mu_{y} \varepsilon_{x} \frac{\partial \phi_{m}}{\partial y}-\omega^{2} \mu_{y} \varepsilon_{x} F_{y}+\left(1-\frac{\varepsilon_{x}}{\varepsilon_{z}}\right) \frac{\partial^{2} F_{y}}{\partial x^{2}}\right] \\
& +\hat{a}_{z} j \omega \mu_{z} \varepsilon_{x} \frac{\partial \phi_{m}}{\partial z}
\end{aligned}
$$

and

$$
\nabla^{2} F-\nabla \nabla \cdot F=-\hat{a}_{x} \frac{\partial^{2} F_{y}}{\partial x \partial y}+\hat{a}_{y}\left(\frac{\partial^{2} F_{y}}{\partial x^{2}}+\frac{\partial^{2} F_{y}}{\partial z^{2}}\right)-\hat{a}_{z} \frac{\partial^{2} F_{y}}{\partial z \partial y}
$$

By equating the equations (16) and (17) on $x$ and $z$ components, we can have

$$
\begin{aligned}
\frac{\partial^{2} F_{y}}{\partial x \partial y} & =-j \omega \mu_{x} \varepsilon_{z} \frac{\partial \phi_{m}}{\partial x} \\
\frac{\partial^{2} F_{y}}{\partial z \partial y} & =-j \omega \mu_{z} \varepsilon_{x} \frac{\partial \phi_{m}}{\partial z}
\end{aligned}
$$

By solving the above two equations, we can have

$$
\begin{aligned}
\frac{\partial F_{y}}{\partial y} & =-j \omega \mu_{x} \varepsilon_{z} \phi_{m}+C \\
\frac{\mu_{x}}{\varepsilon_{x}} & =\frac{\mu_{z}}{\varepsilon_{z}}
\end{aligned}
$$

The equation (21) can be seen as a condition required for the $\mathrm{TE}^{y}$ mode to exist. Finally, by equating the $y$ components of equations of (16) and (17) and using equation (21), we can have the scalar wave equation for vector potential component $F_{y}$,

$$
\mu_{x} \frac{\partial^{2} F_{y}}{\partial x^{2}}+\mu_{y} \frac{\partial^{2} F_{y}}{\partial y^{2}}+\mu_{z} \frac{\partial^{2} F_{y}}{\partial z^{2}}=-\omega^{2} \mu_{y} \varepsilon_{x} \mu_{z} F_{y}=-\omega^{2} \mu_{y} \varepsilon_{z} \mu_{x} F_{y}
$$

A concise scalar wave equation for $F_{y}$ is reached and can be used to solve the electromagnetic fields of $\mathrm{TE}^{y}$ modes with adequate boundary conditions. From equations (10), (11), (13), (21), and (22), we can summarize the results for $\mathrm{TE}^{y}$ mode: 


$$
\begin{aligned}
& F=\hat{a}_{y} F_{y}(x, y, z) \\
& \mu_{x} \frac{\partial^{2} F_{y}}{\partial x^{2}}+\mu_{y} \frac{\partial^{2} F_{y}}{\partial y^{2}}+\mu_{z} \frac{\partial^{2} F_{y}}{\partial z^{2}}+\kappa^{2} F_{y}=0, \kappa^{2}=\omega^{2} \mu_{y} \varepsilon_{x} \mu_{z}=\omega^{2} \mu_{y} \varepsilon_{z} \mu_{x} ; \\
& \frac{\mu_{x}}{\varepsilon_{x}}=\frac{\mu_{z}}{\varepsilon_{z}} ; \\
& E_{x}=\frac{1}{\varepsilon_{x}} \frac{\partial F_{y}}{\partial z}, \quad E_{y}=0, \quad E_{z}=-\frac{1}{\varepsilon_{z}} \frac{\partial F_{y}}{\partial x} ; \\
& H_{x}=-j \frac{1}{\omega \mu_{x} \varepsilon_{z}} \frac{\partial^{2} F_{y}}{\partial x \partial y}, \quad H_{y}=j \frac{1}{\omega \mu_{y} \varepsilon_{x}} \frac{\partial^{2} F_{y}}{\partial z^{2}}+j \frac{1}{\omega \mu_{y} \varepsilon_{z}} \frac{\partial^{2} F_{y}}{\partial x^{2}} \\
& H_{z}=-j \frac{1}{\omega \mu_{z} \varepsilon_{x}} \frac{\partial^{2} F_{y}}{\partial z \partial y}
\end{aligned}
$$

For $\mathrm{TE}^{x}$ and $\mathrm{TE}^{z}$ modes, let $F=\hat{a}_{x} F_{x}(x, y, z)$ and $F=$ $\hat{a}_{z} F_{z}(x, y, z)$ respectively. With the similar procedures as those of $\mathrm{TE}^{y}$ mode from equations (10) to (22), we can obtain the results for $\mathrm{TE}^{x}$ and $\mathrm{TE}^{z}$ modes and those equations are put in the appendix for reference.

Next consider the cylindrical coordinate. For the TE to $z$ direction ( $\mathrm{TE}^{z}$ mode), with the similar procedures as those of the rectangular coordinate, the results are summarized as following:

$$
\begin{aligned}
& F=\hat{a}_{z} F_{z}(r, \phi, z) ; \\
& \mu_{r} \frac{1}{r} \frac{\partial}{\partial r}\left(r \frac{\partial F_{z}}{\partial r}\right)+\mu_{\phi} \frac{1}{r^{2}} \frac{\partial^{2} F_{z}}{\partial y^{2}}+\mu_{z} \frac{\partial^{2} F_{z}}{\partial z^{2}}+\kappa^{2} F_{z} \\
& =\mu_{r}\left[\left(\varepsilon_{r}-\varepsilon_{\phi}\right) \frac{\partial}{\partial \phi} \frac{1}{\varepsilon_{r}}+\frac{1}{\varepsilon_{r}} \frac{\partial \varepsilon_{r}}{\partial \phi}\right] \frac{1}{r^{2}} \frac{\partial F_{z}}{\partial \phi} \\
& \kappa^{2}=\omega^{2} \mu_{z} \varepsilon_{\phi} \mu_{r}=\omega^{2} \mu_{z} \varepsilon_{r} \mu_{\phi} ; \\
& \frac{\mu_{r}}{\varepsilon_{r}}=\frac{\mu_{\phi}}{\varepsilon_{\phi}} ; \\
& E_{r}=-\frac{1}{\varepsilon_{r}} \frac{1}{r} \frac{\partial F_{z}}{\partial \phi}, \quad E_{\phi}=\frac{1}{\varepsilon_{\phi}} \frac{\partial F_{z}}{\partial r}, \quad E_{z}=0 \\
& H_{r}=-j \frac{1}{\omega \mu_{r} \varepsilon_{\phi}} \frac{\partial^{2} F_{z}}{\partial r \partial z}, \quad H_{\phi}=-j \frac{1}{\omega \mu_{\phi} \varepsilon_{r}} \frac{1}{r} \frac{\partial^{2} F_{z}}{\partial \phi \partial z}, \\
& H_{z}=j \frac{1}{\omega \mu_{z} \varepsilon_{\phi}} \frac{1}{r} \frac{\partial}{\partial r}\left(r \frac{\partial F_{z}}{\partial r}\right)+j \frac{1}{\omega \mu_{z}} \frac{1}{r^{2}} \frac{\partial}{\partial \phi}\left(\frac{1}{\varepsilon_{r}} \frac{\partial F_{z}}{\partial \phi}\right) .
\end{aligned}
$$


In the above equations of the $\mathrm{TE}^{z}$ mode in the cylindrical coordinate, the relation of permittivity and permeability shows,

$$
\frac{\mu_{r}}{\varepsilon_{r}}=\frac{\mu_{\phi}}{\varepsilon_{\phi}}
$$

By using equations (8) and (9), this equation can be simplified to,

$$
\frac{\mu_{x}}{\varepsilon_{x}}=\frac{\mu_{y}}{\varepsilon_{y}}
$$

which agrees with the result of the $\mathrm{TE}^{z}$ mode in the rectangular coordinate. In addition, there is an extra $\mu_{r}\left[\left(\varepsilon_{r}-\varepsilon_{\phi}\right) \frac{\partial}{\partial \phi} \frac{1}{\varepsilon_{r}}+\frac{1}{\varepsilon_{r}} \frac{\partial \varepsilon_{r}}{\partial \phi}\right] \frac{1}{r^{2}} \frac{\partial F_{z}}{\partial \phi}$ in the scalar wave equation which comes from the fact that the $\varepsilon_{r}$ and $\varepsilon_{\phi}$ are $\phi$ dependent. This extra term can be eliminated if $\varepsilon_{r}$ and $\varepsilon_{\phi}$ are $\phi$ independent or $\varepsilon_{x}=\varepsilon_{y}$ (uniaxial).

\section{(B) TM Modes}

With the similar roles of $D, E, F$, and $\phi_{m}$ for the TE modes, the relations among $B, H, A$, and $\phi_{e}$ can be used to derive the scalar wave equations of $A_{x}, A_{y}$, and $A_{z}$ for $\mathrm{TM}^{x}, \mathrm{TM}^{y}$, and $\mathrm{TM}^{z}$ modes respectively in a biaxial anisotropic medium. By using equations (2), (5), Maxwell's equation

$$
\nabla \times H=j \omega D
$$

and

$$
E=-\nabla \phi_{e}-j \omega A
$$

we can have the results for TM modes by similar procedures as from equations (10) to (22). In the rectangular coordinate for $\mathrm{TM}^{y}$ mode:

$$
\begin{aligned}
& A=\hat{a}_{y} A_{y}(x, y, z) \\
& \varepsilon_{x} \frac{\partial^{2} A_{y}}{\partial x^{2}}+\varepsilon_{y} \frac{\partial^{2} A_{y}}{\partial y^{2}}+\varepsilon_{z} \frac{\partial^{2} A_{y}}{\partial z^{2}}+\kappa^{2} A_{y}=0, \quad \kappa^{2}=\omega^{2} \varepsilon_{y} \varepsilon_{x} \mu_{z}=\omega^{2} \varepsilon_{y} \varepsilon_{z} \mu_{x} \\
& \frac{\mu_{x}}{\varepsilon_{x}}=\frac{\mu_{z}}{\varepsilon_{z}} ; \\
& H_{x}=-\frac{1}{\mu_{x}} \frac{\partial A_{y}}{\partial z}, \quad H_{y}=0, \quad H_{z}=\frac{1}{\mu_{z}} \frac{\partial A_{y}}{\partial x} ; \\
& E_{x}=-j \frac{1}{\omega \varepsilon_{x} \mu_{z}} \frac{\partial^{2} A_{y}}{\partial x \partial y}, \quad E_{y}=j \frac{1}{\omega \varepsilon_{y} \mu_{x}} \frac{\partial^{2} A_{y}}{\partial z^{2}}+j \frac{1}{\omega \varepsilon_{y} \mu_{z}} \frac{\partial^{2} A_{y}}{\partial x^{2}} \\
& E_{z}=-j \frac{1}{\omega \varepsilon_{z} \mu_{x}} \frac{\partial^{2} A_{y}}{\partial z \partial y}
\end{aligned}
$$


The results for $\mathrm{TM}^{x}$ and $\mathrm{TM}^{z}$ modes are listed in appendix for reference.

For the cylindrical coordinate in $\mathrm{TM}^{z}$ mode:

$$
\begin{aligned}
& A=\hat{a}_{z} A_{z}(r, \phi, z) ; \\
& \varepsilon_{r} \frac{1}{r} \frac{\partial}{\partial r}\left(r \frac{\partial A_{z}}{\partial r}\right)+\varepsilon_{\phi} \frac{1}{r^{2}} \frac{\partial^{2} A_{z}}{\partial y^{2}}+\varepsilon_{z} \frac{\partial^{2} A_{z}}{\partial z^{2}}+\kappa^{2} A_{z} \\
& =\varepsilon_{r}\left[\left(\mu_{r}-\mu_{\phi}\right) \frac{\partial}{\partial \phi} \frac{1}{\mu_{r}}+\frac{1}{\mu_{r}} \frac{\partial \mu_{r}}{\partial \phi}\right] \frac{1}{r^{2}} \frac{\partial A_{z}}{\partial \phi}, \\
& \kappa^{2}=\omega^{2} \varepsilon_{z} \varepsilon_{\phi} \mu_{r}=\omega^{2} \varepsilon_{z} \varepsilon_{r} \mu_{\phi} ; \\
& \frac{\mu_{r}}{\varepsilon_{r}}=\frac{\mu_{\phi}}{\varepsilon_{\phi}} ; \\
& H_{r}=\frac{1}{\mu_{r}} \frac{1}{r} \frac{\partial A_{z}}{\partial \phi}, \quad H_{\phi}=-\frac{1}{\mu_{\phi}} \frac{\partial A_{z}}{\partial r}, \quad H_{z}=0 ; \\
& E_{r}=-j \frac{1}{\omega \varepsilon_{r} \mu_{\phi}} \frac{\partial^{2} A_{z}}{\partial r \partial z}, \quad E_{\phi}=-j \frac{1}{\omega \varepsilon_{\phi} \mu_{r}} \frac{1}{r} \frac{\partial^{2} A_{z}}{\partial \phi \partial z}, \\
& E_{z}=j \frac{1}{\omega \varepsilon_{z} \mu_{\phi}} \frac{1}{r} \frac{\partial}{\partial r}\left(r \frac{\partial A_{z}}{\partial r}\right)+j \frac{1}{\omega \varepsilon_{z}} \frac{1}{r^{2}} \frac{\partial}{\partial \phi}\left(\frac{1}{\mu_{r}} \frac{\partial A_{z}}{\partial \phi}\right) .
\end{aligned}
$$

In the above results for the $\mathrm{TM}^{z}$ mode in the cylindrical coordinate, the extra term $\varepsilon_{r}\left[\left(\mu_{r}-\mu_{\phi}\right) \frac{\partial}{\partial \phi} \frac{1}{\mu_{r}}+\frac{1}{\mu_{r}} \frac{\partial \mu_{r}}{\partial \phi}\right] \frac{1}{r^{2}} \frac{\partial A_{z}}{\partial \phi}$ comes from that the $\mu_{r}$ and $\mu_{\phi}$ are $\phi$ dependent which can also be eliminated if $\mu_{r}$ and $\mu_{\phi}$ are $\phi$ independent or $\mu_{x}=\mu_{y}$.

(C) TEM Modes:

The TEM modes can be solved by using either vector potential $F$ or vector potential $A$. Firstly, consider the rectangular coordinate. Starting with the $\mathrm{TEM}^{y}$ mode, where $E_{y}=H_{y}=0$. By using the vector potential $F$ to solve the $\mathrm{TEM}^{y}$ mode, from equations (15), (20), and (21), we have,

$$
H_{y}=-\frac{\partial \phi_{m}}{\partial y}-j \omega F_{y}=\frac{1}{j \omega \mu_{x} \varepsilon_{z}} \frac{\partial^{2} F_{y}}{\partial y^{2}}-j \omega F_{y}=0
$$

then we can have scalar wave equation of $F_{y}$,

$$
\frac{\partial^{2} F_{y}}{\partial y^{2}}+\beta^{2} F_{y}=0 ; \quad \beta^{2}=\omega^{2} \mu_{x} \varepsilon_{z}=\omega^{2} \mu_{z} \varepsilon_{x}
$$


With a similar procedure, we can have the scalar wave equation of $A_{y}$,

$$
\frac{\partial^{2} A_{y}}{\partial y^{2}}+\beta^{2} A_{y}=0 ; \quad \beta^{2}=\omega^{2} \mu_{x} \varepsilon_{z}=\omega^{2} \mu_{z} \varepsilon_{x}
$$

In summary the electric and magnetic fields of $\mathrm{TEM}^{y}$ mode are

$$
\begin{aligned}
& F=\hat{a}_{y} F_{y}(x, y, z) ; \\
& \frac{\partial^{2} F_{y}}{\partial y^{2}}+\beta^{2} F_{y}=0, \quad \beta^{2}=\omega^{2} \mu_{x} \varepsilon_{z}=\omega^{2} \mu_{z} \varepsilon_{x} \\
& \frac{\mu_{x}}{\varepsilon_{x}}=\frac{\mu_{z}}{\varepsilon_{z}} ; \\
& E_{x}=\frac{1}{\varepsilon_{x}} \frac{\partial F_{y}}{\partial z}, \quad E_{y}=0, \quad E_{z}=-\frac{1}{\varepsilon_{z}} \frac{\partial F_{y}}{\partial x} \\
& H_{x}=-j \frac{1}{\omega \mu_{x} \varepsilon_{z}} \frac{\partial^{2} F_{y}}{\partial x \partial y}, H_{y}=0, H_{z}=-j \frac{1}{\omega \mu_{x} \varepsilon_{z}} \frac{\partial^{2} F_{y}}{\partial z \partial y}
\end{aligned}
$$

or

$$
\begin{aligned}
& A=\hat{a}_{y} A_{y}(x, y, z) ; \\
& \frac{\partial^{2} A_{y}}{\partial y^{2}}+\beta^{2} A_{y}=0, \quad \beta^{2}=\omega^{2} \mu_{x} \varepsilon_{z}=\omega^{2} \mu_{z} \varepsilon_{x} \\
& \frac{\mu_{x}}{\varepsilon_{x}}=\frac{\mu_{z}}{\varepsilon_{z}} ; \\
& H_{x}=-\frac{1}{\mu_{x}} \frac{\partial A_{y}}{\partial z}, \quad H_{y}=0, \quad H_{z}=\frac{1}{\mu_{z}} \frac{\partial A_{y}}{\partial x} ; \\
& E_{x}=-j \frac{1}{\omega \mu_{x} \varepsilon_{z}} \frac{\partial^{2} A_{y}}{\partial x \partial y}, E_{y}=0, E_{z}=-j \frac{1}{\omega \mu_{x} \varepsilon_{z}} \frac{\partial^{2} A_{y}}{\partial z \partial y}
\end{aligned}
$$

The results for $\mathrm{TEM}^{x}$ and $\mathrm{TEM}^{z}$ modes are also in the appendix for reference.

In the cylindrical coordinate for $\mathrm{TEM}^{z}$ mode: 


$$
\begin{aligned}
& F=\hat{a}_{z} F_{z}(r, \phi, z) ; \\
& \frac{\partial^{2} F_{z}}{\partial z^{2}}+\beta^{2} F_{z}=0, \quad \beta^{2}=\omega^{2} \mu_{r} \varepsilon_{\phi}=\omega^{2} \mu_{\phi} \varepsilon_{r} ; \\
& \frac{\mu_{r}}{\varepsilon_{r}}=\frac{\mu_{\phi}}{\varepsilon_{\phi}} \\
& E_{r}=-\frac{1}{\varepsilon_{r}} \frac{1}{r} \frac{\partial F_{z}}{\partial \phi}, \quad E_{\phi}=\frac{1}{\varepsilon_{\phi}} \frac{\partial F_{z}}{\partial r}, \quad E_{z}=0 ; \\
& H_{r}=-j \frac{1}{\omega \mu_{r} \varepsilon_{\phi}} \frac{\partial^{2} F_{z}}{\partial r \partial z}, H_{\phi}=-j \frac{1}{\omega \mu_{\phi} \varepsilon_{r}} \frac{1}{r} \frac{\partial^{2} F_{z}}{\partial \phi \partial z}, H_{z}=0 .
\end{aligned}
$$

or

$$
\begin{aligned}
& A=\hat{a}_{z} A_{z}(r, \phi, z) ; \\
& \frac{\partial^{2} A_{z}}{\partial z^{2}}+\beta^{2} A_{z}=0, \quad \beta^{2}=\omega^{2} \mu_{r} \varepsilon_{\phi}=\omega^{2} \mu_{\phi} \varepsilon_{r} ; \\
& \frac{\mu_{r}}{\varepsilon_{r}}=\frac{\mu_{\phi}}{\varepsilon_{\phi}} ; \\
& H_{r}=\frac{1}{\mu_{r}} \frac{1}{r} \frac{\partial A_{z}}{\partial \phi}, \quad H_{\phi}=-\frac{1}{\mu_{\phi}} \frac{\partial A_{z}}{\partial r}, \quad H_{z}=0 ; \\
& E_{r}=-j \frac{1}{\omega \varepsilon_{r} \mu_{\phi}} \frac{\partial^{2} A_{z}}{\partial r \partial z}, E_{\phi}=-j \frac{1}{\omega \varepsilon_{\phi} \mu_{r}} \frac{1}{r} \frac{\partial^{2} A_{z}}{\partial \phi \partial z}, E_{z}=0 .
\end{aligned}
$$

\section{SUMMARY}

This investigation presents the method for solving the field configurations of TE, TM, and TEM modes for an biaxial anisotropic medium. The concise scalar wave equations of vector potential for various modes and directions are obtained in both rectangular and cylindrical coordinates. Compared to those scalar wave equations of the rectangular coordinate, for the cylindrical coordinate, the scalar wave equations of vector potentials $F_{z}$ and $A_{z}$ for both $\mathrm{TE}^{z}$ and $\mathrm{TM}^{z}$ modes display an extra term, from the $\phi$ dependent of permittivity and permeability respectively. Those extra terms can be eliminated in a uniaxial medium. By solving those scalar wave equations, the electric and magnetic fields of various modes can be obtained by adding adequate boundary conditions. The existence of TE, TM, and TEM modes is clarified. The relationship between permittivity and permeability is critical factor for the existence of desired modes. 


\section{APPENDIX A.}

1. The equations for $\mathrm{TE}^{x}$ and $\mathrm{TE}^{z}$ modes in the rectangular coordinate:

For $\mathrm{TE}^{x}$ mode:

$$
\begin{aligned}
& F=\hat{a}_{x} F_{x}(x, y, z) \\
& \mu_{x} \frac{\partial^{2} F_{x}}{\partial x^{2}}+\mu_{y} \frac{\partial^{2} F_{x}}{\partial y^{2}}+\mu_{z} \frac{\partial^{2} F_{x}}{\partial z^{2}}+\kappa^{2} F_{x}=0, \kappa^{2}=\omega^{2} \mu_{x} \varepsilon_{y} \mu_{z}=\omega^{2} \mu_{x} \varepsilon_{z} \mu_{y} ; \\
& \frac{\mu_{y}}{\varepsilon_{y}}=\frac{\mu_{z}}{\varepsilon_{z}} ; \\
& E_{x}=0, \quad E_{y}=-\frac{1}{\varepsilon_{y}} \frac{\partial F_{x}}{\partial z}, \quad E_{z}=\frac{1}{\varepsilon_{z}} \frac{\partial F_{x}}{\partial y} ; \\
& H_{x}=j \frac{1}{\omega \mu_{x} \varepsilon_{z}} \frac{\partial^{2} F_{x}}{\partial y^{2}}+j \frac{1}{\omega \mu_{x} \varepsilon_{y}} \frac{\partial^{2} F_{x}}{\partial z^{2}}, \quad H_{y}=-j \frac{1}{\omega \mu_{y} \varepsilon_{z}} \frac{\partial^{2} F_{x}}{\partial y \partial x} \\
& H_{z}=-j \frac{1}{\omega \mu_{z} \varepsilon_{y}} \frac{\partial^{2} F_{x}}{\partial z \partial x}
\end{aligned}
$$

For $\mathrm{TE}^{z}$ mode:

$$
\begin{aligned}
& F=\hat{a}_{z} F_{z}(x, y, z) \\
& \mu_{x} \frac{\partial^{2} F_{z}}{\partial x^{2}}+\mu_{y} \frac{\partial^{2} F_{z}}{\partial y^{2}}+\mu_{z} \frac{\partial^{2} F_{z}}{\partial z^{2}}+\kappa^{2} F_{z}=0, \kappa^{2}=\omega^{2} \mu_{z} \varepsilon_{x} \mu_{y}=\omega^{2} \mu_{z} \varepsilon_{y} \mu_{x} \\
& \frac{\mu_{x}}{\varepsilon_{x}}=\frac{\mu_{y}}{\varepsilon_{y}} ; \\
& E_{x}=-\frac{1}{\varepsilon_{x}} \frac{\partial F_{z}}{\partial y}, \quad E_{y}=\frac{1}{\varepsilon_{y}} \frac{\partial F_{z}}{\partial x}, \quad E_{z}=0 \\
& H_{x}=-j \frac{1}{\omega \mu_{x} \varepsilon_{y}} \frac{\partial^{2} F_{z}}{\partial x \partial z}, \quad H_{y}=-j \frac{1}{\omega \mu_{y} \varepsilon_{x}} \frac{\partial^{2} F_{z}}{\partial y \partial z} \\
& H_{z}=j \frac{1}{\omega \mu_{z} \varepsilon_{y}} \frac{\partial^{2} F_{z}}{\partial x}+j \frac{1}{\omega \mu_{z} \varepsilon_{x}} \frac{\partial^{2} F_{z}}{\partial y^{2}}
\end{aligned}
$$


2. The equations for $\mathrm{TM}^{x}$ and $\mathrm{TM}^{z}$ modes in the rectangular coordinate:

For $\mathrm{TM}^{x}$ mode:

$$
\begin{aligned}
& A=\hat{a}_{x} A_{x}(x, y, z) \\
& \varepsilon_{x} \frac{\partial^{2} A_{x}}{\partial x^{2}}+\varepsilon_{y} \frac{\partial^{2} A_{x}}{\partial y^{2}}+\varepsilon_{z} \frac{\partial^{2} A_{x}}{\partial z^{2}}+\kappa^{2} A_{x}=0, \quad \kappa^{2}=\omega^{2} \varepsilon_{x} \varepsilon_{y} \mu_{z}=\omega^{2} \varepsilon_{x} \varepsilon_{z} \mu_{y} \\
& \frac{\mu_{y}}{\varepsilon_{y}}=\frac{\mu_{z}}{\varepsilon_{z}} \\
& H_{x}=0, \quad H_{y}=\frac{1}{\mu_{y}} \frac{\partial A_{x}}{\partial z}, \quad H_{z}=-\frac{1}{\mu_{z}} \frac{\partial A_{x}}{\partial y} ; \\
& E_{x}=j \frac{1}{\omega \varepsilon_{x} \mu_{z}} \frac{\partial^{2} A_{x}}{\partial y^{2}}+j \frac{1}{\omega \varepsilon_{x} \mu_{y}} \frac{\partial^{2} A_{x}}{\partial z^{2}}, \quad E_{y}=-j \frac{1}{\omega \varepsilon_{y} \mu_{z}} \frac{\partial^{2} A_{x}}{\partial y \partial x} \\
& E_{z}=-j \frac{1}{\omega \varepsilon_{z} \mu_{y}} \frac{\partial^{2} A_{x}}{\partial z \partial x}
\end{aligned}
$$

For $\mathrm{TM}^{z}$ mode:

$$
\begin{aligned}
& A=\hat{a}_{z} A_{z}(x, y, z) \\
& \varepsilon_{x} \frac{\partial^{2} A_{z}}{\partial x^{2}}+\varepsilon_{y} \frac{\partial^{2} A_{z}}{\partial y^{2}}+\varepsilon_{z} \frac{\partial^{2} A_{z}}{\partial z^{2}}+\kappa^{2} A_{z}=0, \quad \kappa^{2}=\omega^{2} \varepsilon_{z} \varepsilon_{x} \mu_{y}=\omega^{2} \varepsilon_{z} \varepsilon_{y} \mu_{x} \\
& \frac{\mu_{x}}{\varepsilon_{x}}=\frac{\mu_{y}}{\varepsilon_{y}} ; \\
& H_{x}=\frac{1}{\mu_{x}} \frac{\partial A_{z}}{\partial y}, \quad H_{y}=-\frac{1}{\mu_{y}} \frac{\partial A_{z}}{\partial x}, \quad H_{z}=0 \\
& E_{x}=-j \frac{1}{\omega \varepsilon_{x} \mu_{y}} \frac{\partial^{2} A_{z}}{\partial x \partial z}, \quad E_{y}=-j \frac{1}{\omega \varepsilon_{y} \mu_{x}} \frac{\partial^{2} A_{z}}{\partial y \partial z} \\
& E_{z}=j \frac{1}{\omega \varepsilon_{z} \mu_{y}} \frac{\partial^{2} A_{z}}{\partial x^{2}}+j \frac{1}{\omega \varepsilon_{z} \mu_{x}} \frac{\partial^{2} A_{z}}{\partial y^{2}}
\end{aligned}
$$


3. The equations for $\mathrm{TEM}^{x}$ and $\mathrm{TEM}^{z}$ modes in the rectangular coordinate:

For TEM $^{x}$ mode:

$$
\begin{aligned}
& F=\hat{a}_{x} F_{x}(x, y, z) ; \\
& \frac{\partial^{2} F_{x}}{\partial x^{2}}+\beta^{2} F_{x}=0, \quad \beta^{2}=\omega^{2} \mu_{y} \varepsilon_{z}=\omega^{2} \mu_{z} \varepsilon_{y} \\
& \frac{\mu_{y}}{\varepsilon_{y}}=\frac{\mu_{z}}{\varepsilon_{z}} ; \\
& E_{x}=0, \quad E_{y}=-\frac{1}{\varepsilon_{y}} \frac{\partial F_{x}}{\partial z}, \quad E_{z}=\frac{1}{\varepsilon_{z}} \frac{\partial F_{x}}{\partial y} \\
& H_{x}=0, \quad H_{y}=-j \frac{1}{\omega \mu_{y} \varepsilon_{z}} \frac{\partial^{2} F_{x}}{\partial y \partial x}, H_{z}=-j \frac{1}{\omega \mu_{y} \varepsilon_{z}} \frac{\partial^{2} F_{x}}{\partial z \partial x}
\end{aligned}
$$

or

$$
\begin{aligned}
& A=\hat{a}_{x} A_{x}(x, y, z) ; \\
& \frac{\partial^{2} A_{x}}{\partial x^{2}}+\beta^{2} A_{x}=0, \quad \beta^{2}=\omega^{2} \mu_{y} \varepsilon_{z}=\omega^{2} \mu_{z} \varepsilon_{y} ; \\
& \frac{\mu_{y}}{\varepsilon_{y}}=\frac{\mu_{z}}{\varepsilon_{z}} ; \\
& H_{x}=0, \quad H_{y}=\frac{1}{\mu_{y}} \frac{\partial A_{x}}{\partial z}, \quad H_{z}=-\frac{1}{\mu_{z}} \frac{\partial A_{x}}{\partial y} ; \\
& E_{x}=0, \quad E_{y}=-j \frac{1}{\omega \mu_{y} \varepsilon_{z}} \frac{\partial^{2} A_{x}}{\partial y \partial x}, E_{z}=-j \frac{1}{\omega \mu_{y} \varepsilon_{z}} \frac{\partial^{2} A_{x}}{\partial z \partial x} .
\end{aligned}
$$

For $\mathrm{TEM}^{z}$ mode:

$$
\begin{aligned}
& F=\hat{a}_{z} F_{z}(x, y, z) ; \\
& \frac{\partial^{2} F_{z}}{\partial z^{2}}+\beta^{2} F_{z}=0, \quad \beta^{2}=\omega^{2} \mu_{x} \varepsilon_{y}=\omega^{2} \mu_{y} \varepsilon_{x} \\
& \frac{\mu_{x}}{\varepsilon_{x}}=\frac{\mu_{y}}{\varepsilon_{y}} ; \\
& E_{x}=-\frac{1}{\varepsilon_{x}} \frac{\partial F_{z}}{\partial y}, \quad E_{y}=\frac{1}{\varepsilon_{y}} \frac{\partial F_{x}}{\partial x}, \quad E_{z}=0 \\
& H_{x}=-j \frac{1}{\omega \mu_{x} \varepsilon_{y}} \frac{\partial^{2} F_{z}}{\partial x \partial z}, H_{y}=-j \frac{1}{\omega \mu_{x} \varepsilon_{y}} \frac{\partial^{2} F_{z}}{\partial y \partial z}, H_{z}=0 .
\end{aligned}
$$


or

$$
\begin{aligned}
& A=\hat{a}_{z} A_{z}(x, y, z) \\
& \frac{\partial^{2} A_{z}}{\partial z^{2}}+\beta^{2} A_{z}=0, \quad \beta^{2}=\omega^{2} \mu_{x} \varepsilon_{y}=\omega^{2} \mu_{y} \varepsilon_{x} \\
& \frac{\mu_{x}}{\varepsilon_{x}}=\frac{\mu_{y}}{\varepsilon_{y}} ; \\
& H_{x}=\frac{1}{\mu_{x}} \frac{\partial A_{z}}{\partial y}, \quad H_{y}=-\frac{1}{\mu_{y}} \frac{\partial A_{z}}{\partial x}, \quad H_{z}=0 ; \\
& E_{x}=-j \frac{1}{\omega \mu_{x} \varepsilon_{y}} \frac{\partial^{2} A_{z}}{\partial x \partial z}, E_{y}=-j \frac{1}{\omega \mu_{x} \varepsilon_{y}} \frac{\partial^{2} A_{z}}{\partial y \partial z}, E_{z}=0
\end{aligned}
$$

\section{ACKNOWLEDGMENT}

This work was partially supported by the National Science Council of the Republic of China, Taiwan under Contract No. NSC92-2213-E161-003.

\section{REFERENCES}

1. Moss, C. D., F. L. Teixeira, and J. A. Kong, "Analysis and compensation of numerical dispersion in the FDTD method for layered, anisotropic media," IEEE Trans. Antennas Propagat., Vol. 50, 1174-1184, Sep. 2002.

2. Barrowes, B. E., C. O. Ao, F. L. Teixeira, and J. A. Kong, "Sparse matrix/canonical grid method applied to 3-D dense medium simulations," IEEE Trans. Antennas Propagat., Vol. 51, 48-58, Jan. 2003.

3. Krowne, C. M., "Spectral-domain determination of the propagation constant in biaxial planar media," Int. J. Electronics, Vol. 59, $315-332,1985$.

4. Van Trier, A. A. and M. Th, "Guided electromagnetic waves in anisotropic media," Appl. Sci. Res. (Section B), Vol. 3, 305-371, 1953.

5. Aknin, N., A. El Moussaoui, and M. Essaaidi, "Rigorous analysis of shielded uniaxial anisotropic DRs by the mode-matching method," Microwave Opt. Technol. Lett., Vol. 19, 233-238, June 1995. 
6. Palanichamy, S., A. Biswas, and R. K. Shukla, "Full-wave analysis of dominant and higher order modes in microstrip-slot coupled line with uniaxial anisotropic dielectric substrates," Microwave Opt. Technol. Lett., Vol. 25, 400-406, June 2000.

7. Liu, S., L. W. Li, M. S. Leong, and T. S. Yeo, "Field representation in general rotationally uniaxial anisotropic media using spherical vector wave functions," Microwave Opt. Technol. Lett., Vol. 25, 159-162, May 2000.

8. Li, L. W., S. H. Liu, M. S. Leong, and T. S. Yeo, "Circular cylindrical waveguide filled with uniaxial anisotropic mediaelectromagnetic fields and dyadic Green's functions," IEEE Trans. Microwave Theory Tech., Vol. 49, 1361-1364, July 2001.

9. Wang, C. and K. A. Zaki, "Generalized multiplayer anisotropic dielectric resonators," IEEE Trans. Microwave Theory Tech., Vol. 48, 60-66, Jan. 2001.

10. Losada, V., R. R. Boix, and M. Horno, "Full-wave analysis of circular microstrip resonators in multilayered media containing uniaxial anisotropic dielectrics, magnetized ferrites, and chiral materials," IEEE Trans. Microwave Theory Tech., Vol. 48, 10571064, June 2000.

11. Lindell, I. V. and F. Olyslager, "Decomposition of electromagnetic sources in axially chiral uniaxial anisotropic media," J. Electromagnetic Waves Applications, Vol. 10, 51-59, 1996.

12. Olyslager, F. and D. D. Zutter, "Rigorous boundary integral equation solution for general isotropic and uniaxial anisotropic dielectric waveguides in multilayered media including losses, gain and leakage," IEEE Trans. Microwave Theory Tech., Vol. 41, 1385-1392, Aug. 1993.

13. Shin, S. C. and C. S. Kim, "A new method for analyzing torque curves of uniaxial anisotropic materials," IEEE Trans. Magnetics, Vol. 27, 4852-4854, Nov. 1991.

14. Lindell, I. V., "TE/TM decomposition of electromagnetic sources in uniaxial anisotropic media," Microwave Opt. Technol. Lett., Vol. 19, 233-238, June 1995.

15. Balanis, C. A., Advanced Engineering Electromagnetics, Chap. 6, New York, John Wiley \& Sons, 1989.

Jyh Sheen was born in Taiwan on September 23, 1962. He received the B.S. degree in electrophysics from the National Chiao Tung University, Taiwan, in 1984, and the M.S. and Ph.D. degrees in electrical engineering from the New Jersey Institute of Technology in 
1989 and The Pennsylvania State University in 1993, respectively. In 1994-2001, he was the associate professor of the ChienKuo Institute of Technology, Taiwan. In 2001, he joined the faculty of Oriental Institute of Technology, where he is currently Associate Professor of Communication Engineering Department. His current research interests include the measurements of microwave dielectric properties, microwave circuits, and propagation of electromagnetic wave. 